\title{
Advance care planning in stroke: influence of time on engagement in the process
}

\author{
This article was published in the following Dove Press journal: \\ Patient Preference and Adherence \\ 24 January 2014 \\ Number of times this article has been viewed
}

\author{
Theresa Green' \\ Shreyas Gandhi ${ }^{2}$ \\ Tessa Kleissen' \\ Jessica Simon ${ }^{1,3}$ \\ Shelley Raffin-Bouchal' \\ Karla Ryckborst ${ }^{4}$ \\ 'Faculty of Nursing, University \\ of Calgary, Calgary, AB, Canada; \\ ${ }^{2}$ Health Sciences, McMaster \\ University, Hamilton, ON, Canada; \\ ${ }^{3}$ Department of Medicine, University \\ of Calgary, Calgary, AB, Canada; \\ ${ }^{4}$ Calgary Stroke Program, Alberta \\ Health Services, Calgary, AB, Canada
}

Correspondence: Theresa Green University of Calgary, Professional Faculties Building, 2500 University Drive NW, Calgary, AB T2N IN4, Canada $\mathrm{Tel}+$ I 4032202464

Fax +I 4032109838

Email greentl@ucalgary.ca
Purpose: Individuals who experience stroke have a higher likelihood of subsequent stroke events, making it imperative to plan for future medical care. In the event of a further serious health event, engaging in the process of advanced care planning (ACP) can help family members and health care professionals (HCPs) make medical decisions for individuals who have lost the capacity to do so. Few studies have explored the views and experiences of patients with stroke about discussing their wishes and preferences for future medical events, and the extent to which stroke HCPs engage in conversations around planning for such events. In this study, we sought to understand how the process of ACP unfolded between HCPs and patients post-stroke.

Patients and methods: Using grounded theory (GT) methodology, we engaged in direct observation of HCP and patient interactions on an acute stroke unit and two stroke rehabilitation units. Using semi-structured interviews, 14 patients and four HCPs were interviewed directly about the ACP process.

Results: We found that open and continual ACP conversations were not taking place, patients experienced an apparent lack of urgency to engage in ACP, and HCPs were uncomfortable initiating ACP conversations due to the sensitive nature of the topic.

Conclusion: In this study, we identified lack of engagement in ACP post-stroke, attributable to patient and HCP factors. This encourages us to look further into the process of ACP in order to develop open communication between the patient with stroke, their families, and stroke HCPs.

Keywords: qualitative, engagement, health care providers, palliative

\section{Introduction}

Stroke is a disease with high levels of morbidity and mortality. One consequence of stroke for patients with significant comorbidities (eg, diabetes, hypertension, cardiac disease) is the increased potential for subsequent stroke events; ${ }^{1-3}$ fear of a recurrent stroke has been identified as a major concern for patients and family. ${ }^{4-6}$ Patients are often unsure about recovery and the possibility of dying from stroke and may wish to face this uncertainty and to address end-of-life issues with the help of health care professionals (HCPs). ${ }^{7,8}$ Researchers studying end-of-life care in patients with acute stroke have reported that issues that are consistently raised include the wish to be involved in their own medical decisions and management, communication, and to obtain information about stroke, components of the advanced care planning (ACP) process. ${ }^{8}$ These issues prompted us to question: 1) the preparedness of stroke patients and families to make important decisions regarding treatment (or lack of) for subsequent health events, and 2) the extent to which HCPs engage in conversations with stroke patients around ACP. 


\section{Purpose}

The aim of this study was to explore how the process of engaging in ACP conversations unfolded between patients and HCPs following stroke, and how perceptions, individual characteristics, and social factors influenced patient engagement in ACP decision making. We anticipated that patients would be interested in ACP in the weeks following their stroke and that HCPs would be hesitant to engage in ACP conversations. The consolidated criteria for reporting qualitative research (COREQ) ${ }^{9}$ was used to guide this report of the research (see Table S1 for COREQ checklist).

\section{Background $\mathrm{ACP}$}

The Canadian Hospice Palliative Care Association summarizes $\mathrm{ACP}$ as " ... the development and expression of wishes for the goals of medical treatment and the continuation or discontinuation of such treatment and care. It involves ongoing discussions with family and friends with whom the person has a relationship, and may involve health care providers, and/or lawyers who may prepare wills and powers of attorney. Advance care planning also involves naming a substitute decision maker."10 Through the ACP process, individuals can identify their preferences in relation to care they will receive if they become incapacitated and unable to make decisions. ${ }^{11,12}$ ACP offers the potential to improve patient and family experiences post-stroke, with increased likelihood that patients' wishes will be respected and better family outcomes. ${ }^{13-15}$

\section{Barriers to ACP}

There is clear evidence regarding potential barriers to ACP. In identifying issues related to chronic obstructive lung disease, major obstacles to $\mathrm{ACP}$ included inadequate information provided at time of diagnosis; lack of consensus about who should initiate ACP conversations and where they should occur; and lack of understanding of the meaning of end of life within the context of chronic obstructive pulmonary disease. ${ }^{13}$ Fried et al ${ }^{11}$ identified barriers that included lack of knowledge about ACP, difficulty thinking about dying, an inability to plan for the future, a belief that family members would know what to do, a belief that their physician would make decisions or that there may not be any choices to be made, and a belief that putting things in writing may actually influence withdrawal of medical treatment.

While there is increasing recognition that $\mathrm{ACP}$ is an important part of health care services, specific interactions with patient and family are not routinely undertaken by most
HCPs. ${ }^{16,17}$ Patients described conversations with physician/ HCPs as lacking in key areas, including emotional support, communication about prognosis or what dying might look like, and spirituality/religion. ${ }^{13}$ The presentation of prognostic medical information is evidently important in changing goals of treatment for patients, and if the information is not presented in an appropriate manner, patients may not change their goals of care to fit their current needs. ${ }^{18}$

While there is evidence supporting ACP in the primary care setting, little is known about what ACP looks like in an acute care environment. For the stroke population, engaging in therapeutic conversations about ACP following stroke may be challenging, as symptoms, patient and family needs, and the context of service delivery will be different for those in different stages of recovery (eg, acute versus chronic stage). ${ }^{8}$ It remains unknown whether patients with mild or severe stroke receive adequate ACP support from HCPs. While the Canadian Best Practice Recommendations for Stroke Care ${ }^{19}$ identify that ACP is necessary, to date, little is known about how patients and HCPs view ACP. In this qualitative study, we attempted to address these issues around $\mathrm{ACP}$ conversations during the acute and rehabilitative phase of stroke.

\section{Methods}

Qualitative grounded theory (GT) was used to investigate how patients/families and HCPs experience the process of ACP following acute stroke. Study findings (the generated theoretical model) will form the basis for a subsequent targeted intervention strategy. Charmaz $^{20}$ describes GT as a "method enabling researchers to explore ideas in conversations and analyze observations from the field." Themes from ACP conversations were revealed through observation, interviews, and materials gathered related to ACP and patients with stroke.

The Consolidated Criteria for Reporting Qualitative Research (COREQ); were used to ensure quality of the research process (eg, aspects of the research team, study methods, findings, analysis and interpretations). ${ }^{21}$

\section{Participants}

Patient participants were recruited for the study from an acute care stroke unit and two stroke rehabilitation units in a large city in southern Alberta, Canada. Adult ( $\geq 18$ years of age) participants up to 12 weeks post-stroke were approached for recruitment into the study. This time frame allowed us to recruit patients while the stroke experience was still relatively fresh in their minds and to recruit from both inpatient and rehabilitation unit settings. Patients with dysphasia were included depending on the severity of the speech deficit. Patients who were non-English speaking, $<18$ years of 
age, aphasic (impairment of the ability to process language, written and spoken), unconscious, or cognitively impaired (the inability to recall two or more items on a three-item test of short-term recall) were excluded. ${ }^{22}$

The client service managers of the acute stroke unit and two stroke rehabilitation units acted as liaisons to approach potential staff and patient participants. Potential patient participants who indicated a willingness to be approached were contacted by a research assistant (RA) to explain the study, obtain informed consent, and set up a time for the actual interview. HCPs were recruited using snowball sampling; for example, staff who participated in the interview suggested other potential contacts. Individual HCP consent was obtained at the initial meeting and a time/place was identified for the actual interview.

\section{Ethics}

Ethics approval for this study was received from the local Health Ethics Review Board prior to initiating participant recruitment. Informed consent was obtained from each participant after apprising them of the background, purpose, and study procedures; potential risks and benefits; and confidentiality processes. Participants were also informed of their right to refuse participation or to withdraw from the study at any time. As their interview data would be transcribed immediately following the interview and used to inform the next interview, we did indicate their anonymized data would remain in the study.

\section{Data collection and analysis}

The primary methods of data collection were participant observation and audio-taped semi-structured interviews. ${ }^{23-25}$ A semi-structured interview guide was used, with openended questions specifically focused on discovering what the process of having ACP conversations looked and felt like for those involved. The primary author and RAs (female RAs; students and staff) conducted all patient and HCP interviews. The lead investigator has prior experience in conducting qualitative interviews and focus groups, and provided training to the RAs prior to initiating the study. Guidance was also obtained from a senior investigator with experience in ACP.

Observational data were collected and memo-ing (record of observations of people, a situation, or an environment by making notes of what has been observed) performed to preserve ideas and provide an audit trail of abstractions, concept integration, emerging hypotheses, and analytical schemes for theory generation. A questionnaire regarding sociodemographic and health status was collected from participants. These data included age; ethnicity; marital, education, and employment status; co-morbidities; and classification of stroke type. These data allowed for a description of the participant pool and helped direct the theoretical sampling process.

In GT, the theory itself is constructed to explain observed relationships that are tested for validity with participants and other sources as they emerge from the data. ${ }^{26,27}$ Using a constant comparative analytic method, the discussion guide was modified as needed to explore concepts revealed in earlier interviews. Audio-taped interviews and field notes were transcribed (a procedure for producing a written version of the interview) and reviewed with data included in the overall analysis process. Participants were purposively sampled until no new information or codes emerged (data saturation). Data analyses were conducted by the lead researcher and RAs, followed by verification by the co-investigators of themes emerging from the data. The qualitative software NVivo $9^{\text {TM }}$ (QSR International, Melbourne, VIC, Australia) was used to manage the data.

\section{Results}

Stroke survivors ( $n=14$; six males, eight females) aged between 37 and 87 years participated in the study. Of these, four had experienced a prior stroke or transient ischemic attack (TIA). The HCP sample $(n=4)$ consisted of three nurses and one social worker (all females). Each participant and HCP was interviewed for 30-60 minutes in a single semi-structured interview. All interviews were audio-taped except with one participant who felt uncomfortable with the recording. No participants refused to carry out the interviews or dropped out of the study, and all interviews were conducted with individual participants (no family or others) in a private room or office location in the health care facility. Two focus groups were conducted with study patient and HCP participants to present key themes that emerged from the data and to obtain feedback on the findings.

As a result of the interviews with the participants and HCPs, several key themes emerged related to why participants do or do not take part in the process of ACP. These themes included the role of family members, lack of communication with HCPs, timing, and lack of understanding of the ACP process.

\section{ACP conversations and responses Role of family members and comprehension of stroke severity}

The role that patients themselves and/or family had in medical decision making greatly impacted the ACP process, and an understanding of these roles was essential to appropriate ACP. 
The majority of participants did not experience an urgent need to engage in extensive ACP discussions because they were under the impression that their family members, especially their spouse and their doctor, would be able to carry out their wishes should the need arise. Participants held a common assumption that family member(s) would know how to proceed with health care decisions, even in the absence of explicit instructions from the patient.

Interviewer (I): So, have you talked to anybody about what you want to happen in case you were to have another stroke?

Patient $1(\mathrm{P} 1)$ : ... I've talked to my husband

I: Yeah

P1: It's in my husband's hand[s]

I: ... do you think if you were to have another stroke that they would know what you would like to be done?

P2: Yup. Well my husband knows it all.

Another aspect that may have contributed to the lack of urgency was the perceived low amount of information patients recalled receiving about the severity of their stroke. If patients were not aware that their stroke placed them at significant risk for future events, they were unlikely to consider planning for care in the event of another serious illness.

HCP1: I mean it was a very devastating stroke [for the patient] and they were asking the questions about the what if, what happens next and I said, just, I said well as you know this is a very serious stroke. And they said, well nobody's ever said that to us. And I thought well, it's clear to me it's serious; the person was mute, was barely conscious and had definite dense plegia.

In a later conversation the HCP added:

HCP1: ... So they're very intelligent, well-read individuals and I thought, oh my God ... they didn't comprehend the seriousness or the gravity of it [stroke] yet I thought.

\section{Lack of communication with HCPs}

HCPs can be key participants in ACP by encouraging involvement in discussions and sharing prognostic information and the likely outcomes of treatment. These types of ongoing conversations between the patients and HCPs are important in the development of an appropriate medical care plan for the future. However, it was found that ACP conversations were not taking place. As a result, the majority of patients were unaware of what ACP entails and this was reflected in several interviews.
I: ... when you were having your stroke, did any health care professional, um, come up and talk to you about advanced care planning?

P3: Nobody consulted me, no.

HCP4: ... I don't know them well enough to have, in my opinion, to have the conversation [ACP], and again, I don't know this group of patients I'm working with so my time is spent getting to know patients and not to sit down and have a conversation with that family about goals of care, unless they approach directly ... which most families don't, and most patients don't.

There appeared to be a lack of perception on the part of the HCPs of opportune times to take advantage of openings when patients might be receptive to thinking about the issue of ACP. This was compounded by discomfort in approaching the topic with patients - how to start the conversation and how to finish. Discomfort could also be a result of lack of awareness on the part of the HCP about what ACP actually is.

HCP3: ... part of the admission process is to see if they have a directive and what their, specify what they want. I don't really [feel] comfortable going into the whole do you want life support and all that kind of stuff ... [I] ask them if they would like to have a directive, then we can set them up with the Social Worker, who gives them more information. I don't do a lot of that myself. I just kind of pass it on.

Thus it was apparent that many nursing staff felt a discussion about ACP was outside of their practice comfort zone, and indeed there was a lack of awareness of processes (ways of engaging) used to promote ACP. Staff also often felt these were conversations ideally held between the patient and family members and their primary care physician.

\section{Lack of understanding of ACP}

The lack of knowledge about ACP was demonstrated by the fact that some participants considered ACP to be synonymous with an advanced directive, designation of care, or a living will. Patients largely believed that once they assign someone the responsibility to make their health care decisions in case of a debilitating illness, they have everything covered. The next step in the process, after assigning designation of care, such as having explicit conversations about various medical outcomes with physicians and/or family members, is often not taking place.

HCP1: The most we get nowadays which is a power of attorney for decision making. That seems to be about the only discussions. They're getting, families are getting 
better at making sure they've nominated somebody in the event they can't make their own decisions ... but they don't seem to take the next step of communicating to them what their decisions would be and it's more like they'll know what to do.

Furthermore, some patients also shared the belief that engaging in ACP conversations would in some way hinder the medical care that they were receiving or lead to unwanted consequences in the future. There was a sense of susceptibility to further events brought about by talking about their particular situation.

HCP1: ... and I think too, they're very afraid that we're gonna give up on them.

HCP2: ... in many cases there are times where I, when I'm talking about this, a family member says to me, "I don't want that $[\mathrm{ACP}]$ because in, I saw this thing on TV, they put you to sleep without making [an effort]", so there's a lot of misconception about this.

\section{Timing}

A primary focus of most of the participants in the rehabilitation setting was their personal physical recovery experience. This is significant as they were not very concerned about ACP at this point in time. Lack of engagement in ACP was evident in patient attitudes (focus on physical rehabilitation activities) observed by HCPs involved in primary care. It was not clear whether this lack of engagement was attributable to cognitive impairment post-stroke, personal discomfort in thinking or talking about deteriorating health and death, a sense of ACP being irrelevant to them at this time, or a lack of awareness of the ACP process altogether.

HCP1: ... stroke patients is, well it's like they've been hit over the head with a baseball bat, their ability to take in or comprehend in the acute phase, even if they look like they're fairly alert it just isn't there and they don't recall it afterwards, too much going on.

Therefore, timing may have contributed to the general resistance faced when attempting to get the participants to open up about their thoughts around ACP. Since they were heavily focused on their rehabilitation process, participants were generally very hesitant to openly engage in ACP discussions.

P4: ... I've done my best to cooperate fully with the physio and occupational therapy to get myself back, and to, you know, previous because ... anyway, uh, life is a challenge you meet head on.
P5: ... my leg is doing really good and I just finished, finished physio for my leg and probably sometime this afternoon I'll have my hand done again.

HCP1: ... and in rehab it seems like they're focused on what's next, getting back home or getting whatever that they're not, they don't entertain the notion of what if this happens again.

HCP2: ... the people that I talk about, there are a number of clients who will say, once I get home then I'll be ready to have that conversation with my family.

Several HCP participants observed that ACP requires more time and attentiveness from providers than is usual during the provision of patient care, highlighting another barrier to ACP.

\section{Discussion ACP conversations and issues}

In this study, we corroborated findings of other studies regarding the barriers faced when trying to engage patients and HCPs in ACP conversations; specifically, a lack of urgency around engaging in conversations, lack of communication overall, and timing of the actual study interviews. The first major barrier was the lack of urgency to engage in ACP on the part of the patients. Factors contributing to the lack of urgency included the role of family members in care decision making and misunderstanding the severity of the patient's stroke. Patients believed that their family members would be able to undertake making the right decisions in case of a debilitating illness in the future. Davison ${ }^{7}$ identified a similar belief in patients with end-stage renal disease who expressed complete faith in their "loved ones" to carry out their end-of-life wishes. Unfortunately, evidence suggests there is less decisional congruence when patients and families have not engaged in explicit discussions beforehand. ${ }^{10}$ Decisions taken by family regarding the medical care could go against the wishes of the patient or could lead to a difficult decision for family members in the absence of explicit instruction from the patient. ${ }^{28}$

Moreover, the perceived lack of communication with HCPs about the severity of the stroke also contributed to the lack of urgency. A few patients were unaware of their risk of further deterioration or recurrent stoke and their potential to lose capacity for medical decision making. Without this context, patients might not consider the need to engage in $\mathrm{ACP}$ and not understand what kinds of decisions their families might face in the future (eg, tube feeding in the event of dysphagia). Evidence from literature suggests a similar lack of understanding from many patients with chronic 
conditions and that ACP is unlikely to be easily achieved for the population of patients with limited information about aspects of their condition. ${ }^{29}$

The lack of communication with HCPs also led to gaps in patient knowledge about ACP, which might explain the lack of interest in engaging in ACP conversations. The lack of these conversations could also have been a result of HCP discomfort in initiating discussions around the sensitive topic of ACP. Nurses have identified discomfort in addressing ACP due to fear of anticipated reactions on the part of patients and families, as well as how these reactions will affect themselves. ${ }^{16}$ These nurses also reported a lack of confidence in actually starting the ACP conversation, concern with the reactions of colleagues, and worry about divergent cultural beliefs and practices. Hence, a majority of the participants were not aware of the components of ACP, and ongoing conversations with HCPs regarding ACP were not taking place. Engaging in open conversations requires some understanding of how best to address barriers to encourage initiation of these conversations on the part of HCPs.

Finally, timing of ACP conversations was another major issue to consider. Post-stroke patients in our study were primarily focused on their rehabilitation and getting back to full health. Hence, they were not too concerned about planning ahead in the event of another debilitating illness. Fried et $\mathrm{al}^{10}$ identified this lack of interest in terms of the 'stages of readiness' model for the patient, in which the attitudes of the patients regarding ACP need to change before they change their behavior. In post-stroke rehabilitation settings, the majority of our patients were not yet 'ready' to change their behaviour regarding ACP and hence “... suggests a need for assessments and interventions not only tailored to the individual but also developed separately for each of the different behaviors composing the process of ACP.' HCPs need to find an appropriate time to discuss these issues with the patient and to be alert to cues that patients are ready to change their behavior and attitude concerning ACP. In summary, it appears a lack of urgency, lack of communication with HCPs, lack of understanding of ACP, and timing of conversations post-stroke are all factors that may contribute to low engagement with the ACP process.

\section{Limitations and implications for clinical practice}

This preliminary study does suggest a need for more clinical support and training for HCPs regarding ACP conversations. The comfort level regarding engagement in ACP is not very high, and initiation of these conversations requires great skill. Comprehensive training is needed around initiating conversations that are focused, relevant, and tailored to participants' individual needs..$^{29,30}$

Our study design also only allowed one interview with each participant, and follow-up interviews were not conducted. Follow-up interviews could have indicated potential changes in attitudes regarding ACP over time. In particular, it would be useful for the patient discharge form sent to the primary care physician to include a prompt for the physician to address ACP with the patient and family in their post-discharge follow-up visit. Additionally, a conversation around ACP could be factored into acute- and rehabilitation unit-level discharge planning protocols and checklists. Further exploration around engagement (or lack thereof) in ACP from the family perspective will be vital in the development of best practice guidelines for ACP in stroke. Examination of issues such as privacy or cultural beliefs around illness and death may provide information related to the lack of engagement of all parties in ACP discussions. ${ }^{17}$ Finally, participants were recruited from both acute and chronic settings in their post-stroke care, and future studies could explore the differences (if any) in patient attitudes between the two settings.

\section{Conclusion}

In this study, we identified a lack of engagement in ACP by patients in acute care and stroke rehabilitation settings, attributable to both patient and HCP factors. This encourages us to look further into the process in order to open communication between patients, their families and their health care providers.

\section{Acknowledgments}

The authors acknowledge the RAs for their work in conducting the participant interviews, and the participants themselves for providing their thoughts and experiences in the interviews.

\section{Disclosure}

The authors report no conflicts of interest in this work.

\section{References}

1. Röther J, Alberts MJ, Touzé E, et al. Risk factor profile and management of cerebrovascular patients in the REACH registry. Cerebrovasc Dis. 2008;25:366-374.

2. Rothwell PM. Incidence, risk factors and prognosis of stroke and TIA: the need for high-quality, large-scale epidemiological studies and meta-analyses. Cerebrovasc Dis. 2003;16 Suppl 3:2-10.

3. Rothwell PM, Coull AJ, Giles MF, et al. Change in stroke incidence, mortality, case-fatality, severity, and risk factors in Oxfordshire, UK from 1981 to 2004 (Oxford Vascular Study). Lancet. 2004;363:1925-1933. 
4. Green TL, King KM. The trajectory of minor stroke recovery for men and their female spousal caregivers: literature review. J Adv Nurs. 2007:58:517-531.

5. Green TL, King KM. Experiences of male patients and wife-caregivers in the first year post-stroke following minor stroke: a descriptive qualitative study. Int J Nurs Stud. 2009;46:1194-1200.

6. Green TL, King KM. Functional and psychosocial outcomes 1 year after mild stroke. J Stroke Cerebrovasc Dis. 2010;19:10-16.

7. Davison SN. Advance care planning in patients with end-stage renal disease. Prog Palliat Care. 2009;17:170-178.

8. Payne S, Burton C, Addington-Hall J, Jones A. End-of-life issues in acute stroke care: a qualitative study of the experiences and preferences of patients and families. Palliat Med. 2010;24:146-153.

9. Tong A, Sainsbury P, Craig J. Consolidated criteria for reporting qualitative research (COREQ): a 32-item checklist for interviews and focus groups. Int J Qual Health Care. 2007;19(6):349-357.

10. Canadian Hospice Palliative Care Association, Task Group on Advance Care Planning. Advance Care Planning in Canada: National Framework 2012. Canadian Hospice Palliative Care Association. Available from: http://www.advancecareplanning.ca/media/40158/ acp\%20framework\%202012\%20eng.pdf. Accessed May 17, 2013.

11. Fried TR, Bullock K, Iannone L, O’Leary JR. Understanding advance care planning as a process of health behavior change. JAm Geriatr Soc. 2009;57:1547-1555.

12. Wilkinson A, Wenger N, Shugarman LR. Literature Review on Advance Directives. Report no HHS-100-03-0023. Washington, DC: RAND Corporation; 2007. Available from: http://aspe.hhs.gov/daltcp/reports/2007/ advdirlr.htm. Accessed May 17, 2013.

13. Gott M, Gardiner C, Small N, et al. Barriers to advance care planning in chronic obstructive pulmonary disease. Palliat Med. 2009;23: 642-648.

14. Detering KM, Hancock AD, Reade MC, Silverster W. The impact of advance care planning on end of life care in elderly patients: randomized controlled trial. BMJ. 2010;340:c1345.

15. Silveira MJ, Kim S, Langa KM. Advance directives and outcomes of surrogate decision making before death. $N$ Engl J Med. 2010;362: 1211-1218.

16. Ceccarelli CM, Castner D, Haras MS. Advance care planning for patients with chronic kidney disease - why aren’t nurses more involved? Nephrol Nurs J. 2008;35:553-557.
17. Johnstone MJ, Kanitsaki O. Ethics and advance care planning in a culturally diverse society. J Transcult Nurs. 2009;20:405-416.

18. Aldridge M, Barton E. Establishing terminal status in end-of-life discussions. Qual Health Res. 2007;17:908-918.

19. Lindsay MP, Gubitz G, Bayley M, Phillips S, on behalf of the Canadian Stroke Best Practices and Standards Working Group. Canadian Best Practice Recommendations for Stroke Care. 4th ed. Ottawa: Canadian Stroke Network; 2012

20. Charmaz K. Constructing Grounded Theory: A Practical Guide Through Qualitative Analysis. London: Sage Publications Ltd; 2006.

21. Tong A, Sainsbury P, Craig J. Consolidated criteria for reporting qualitative research (COREQ): a 32-item checklist for interviews and focus groups. Int J Qual Health Care. 2007;19(6):349-357. Available from http://www.equator-network.org/reporting-guidelines/consolidatedcriteria-for-reporting-qualitative-research-coreq-a-32-item-checklistfor-interviews-and-focus-groups/. Accessed January 6, 2014.

22. Brodaty H, Low LF, Gibson L, Burns K. What is the best dementia screening instrument for general practitioners to use? Am J Geriatr Psychiatry. 2006;14:391-400.

23. Glaser BG, Strauss AL. The Discovery of Grounded Theory: Strategies for Qualitative Research. Chicago: Aldine Publishing Company; 1967.

24. Spradley JP. Participant Observation. New York: Holt, Rinehart and Winston; 1980.

25. Strauss AC, Corbin JM. Basics of Qualitative Research: Grounded Theory Procedures and Techniques. Newbury Park (CA): Sage Publications Ltd; 1990.

26. Field PA, Morse JM. Nursing Research: The Application of Qualitative Approaches. London: Croom Helm; 1985

27. Morse JM, Field PA. Qualitative Research Methods for Health Professionals. Thousand Oaks (CA): Sage Publications Ltd; 1995.

28. Dizon DS, Schutzer ME, Politi MC, Linkletter CD, Miller SC, Clark MA, et al. Advance care planning decisions of women with cancer: provider recognition and stability of choices. J Psychosoc Oncol. 2009;27: 383-395.

29. Barnes S, Gardiner C, Gott M, et al. Enhancing patient-professional communication about end-of-life issues in life-limiting conditions: a critical review of the literature. J Pain Symptom Manage. 2012;44: 866-879.

30. Simon J, Murray A, Raffin S. Facilitated advance care planning: what is the patient experience? J Palliat Care. 2008;24:256-264. 


\section{Supplementary material}

Table SI COREQ criteria'

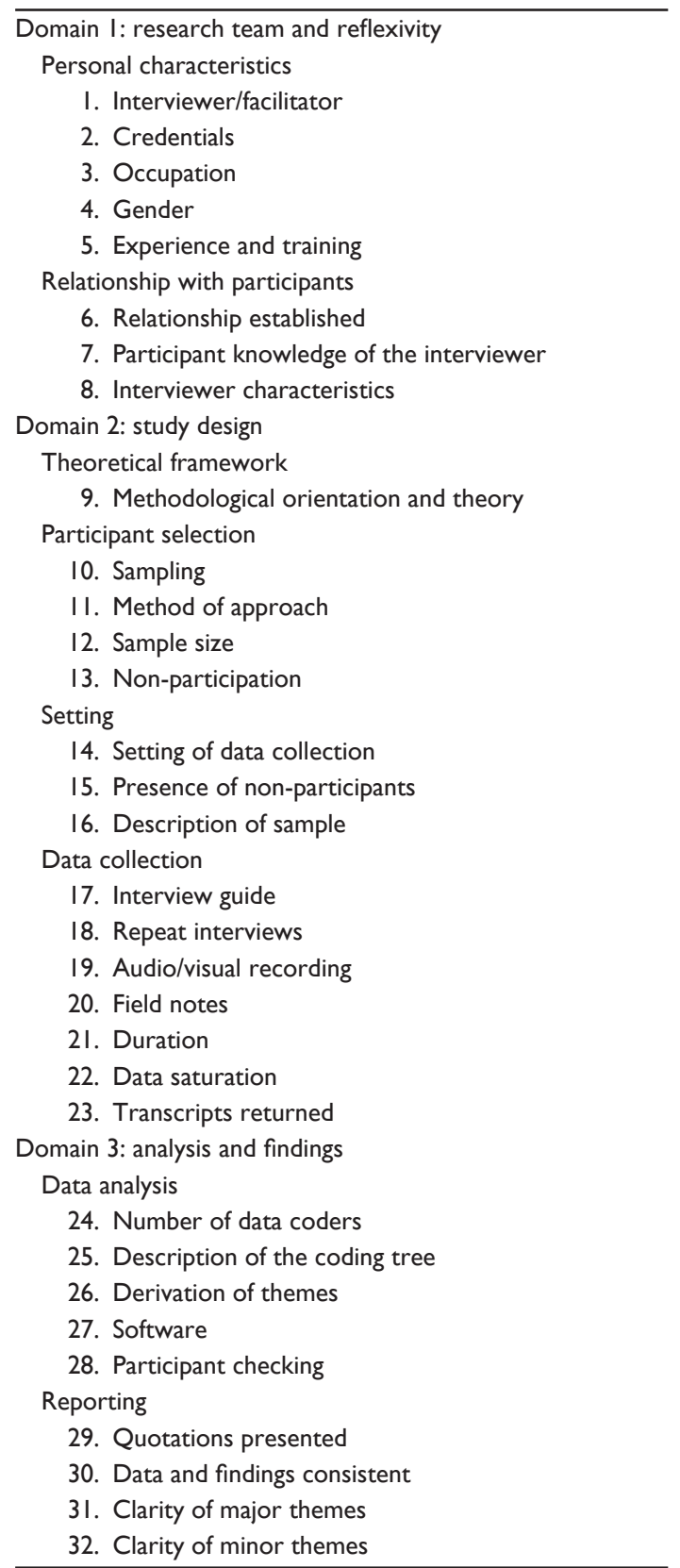

Notes: Adapted from Tong A, Sainsbury P, Craig J. Consolidated criteria for reporting qualitative research (COREQ): a 32-item chicklist for interviews and focus groups. Int J Qual Health Care. 2007;19(16):349-357, by permission of Oxford University Press.'

Abbreviation: COREQ, consolidated criteria for reporting qualitative research.

Patient Preference and Adherence

\section{Publish your work in this journal}

Patient Preference and Adherence is an international, peer-reviewed, open access journal focusing on the growing importance of patient preference and adherence throughout the therapeutic continuum. Patient satisfaction, acceptability, quality of life, compliance, persistence and their role in developing new therapeutic modalities and compounds to

\section{Reference}

1. Tong A, Sainsbury P, Craig J. Consolidated criteria for reporting qualitative research (COREQ): a 32-item chicklist for interviews and focus groups. Int J Qual Health Care. 2007;19(16):349-357. optimize clinical outcomes for existing disease states are major areas of interest. This journal has been accepted for indexing on PubMed Central. The manuscript management system is completely online and includes a very quick and fair peer-review system. Visit http://www.dovepress.com/ testimonials.php to read real quotes from published authors. 